it is therefore possible first to determine the amount of this material present and then, by staining, to ascertain by what process it has been prepared.

This work has more recently been taken up and extended in a series of publications by B. Schulze and E. Göthel ${ }^{3}$. They also examined a large number of dyes and confirm the suitability of rhodamine and eosin, and in addition, recommend sulphorhodamine$G$ and certain of the flavophosphines; the former may be used to distinguish unbleached soda pulp from bleached soda and sulphite pulps, in the absence of unbleached sulphite pulp. Brilliant dianil green was also found to be a useful aid to the distinction of summer and spring woods, with which it gives a blue and yellow colour, respectively. Adansonia (light blue) could be differentiated from manilla and jute, and diagnostic details of other unusual fibres such as Gampi, Mitsumata and Kodzu were also obtained; in some cases these methods have been developed quantitatively. A word of caution seems desirable in connexion with the use of rhodamine6- $G$ for fluorescence experiments, as the writer has found that dyes from different sources sold under this name may give entirely different effects; this indeed may explain the failure of Schulze and Gothel to confirm some of the results of Noss and Sadler where this dye has been involved.

Reference should also be made to the work of $\mathbf{E}$. Grunsteidl ${ }^{4}$ in which similar principles are applied to textile fibres. The primary fluorescence of such fibres, that is, the fluorescence obtained in the unstained state, is itself of value for diagnostic purposes, raw cotton being bright blue (dull grey if mercerised), whilst various silks (real and 'artificial') may be differentiated if examined under controlled conditions. The use of dyes and stains, however, and in particular of quinosol followed by alkali, has proved an additional aid; for example, linen then appears yellow and cotton violet. Similarly $\mathbf{A}$. Segitz ${ }^{5}$ records the use of extract of spruce bark for the differentiation of materials of the "Cellophane" type.

These examples are necessarily restricted in use and as yet incompletely investigated, but they at least serve to illustrate the possibilities awaiting investigation in this field.

J. G.

\section{NATURE, 133, 124, Jan. 27, 1934.}

2 Pap. Fabrikant, 31, 413 ; 1933. Korn, ibid., 32, 181; 1934

${ }^{3}$ ibid., 32, 110; 1934. Zellstoff Pap., 14, 93; 1934. Woch. Pap.

No. $7 ; 1934$

Faserforschung, 10, 215 ; 1933. Rayon and Melliand Text. Month.

, 88,$93 ; 1934$

Pap. Fabrikant, 28, 206; 1930.

\title{
Microplankton and Hydrography of the Great Barrier Reef*
}

$\mathrm{M}^{1}$ ISS S. M. MARSHALL, in her contribution to the Scientific Reports of the Great Barrier Reef Expedition, describes the production of microplankton in the Great Barrier Reef throughout the year. This is the first time that an opportunity has occurred of obtaining continuous observations in one place for so long a time in these regions. The results of the work carried on in the lagoon of the Great Barrier Reef enables a comparison to be made between the conditions in the tropies and those in temperate waters. The water samples used were from various depths, forming part of the routine work at the plankton and hydrographic stations taken by the Nansen-Petterson water bottle. Outside samples were also taken with a glass sample bottle. The material was centrifuged and counted (100-200 c.c. of each sample). Most of it was examined when fresh, thus allowing the small naked dinoflagellates and coccolithophores to be included; the remainder was preserved in strong Fleming solution.

The organisms were grouped so far as possible in their genera. Neritic forms are predominant as was to be expected, especially among the diatoms, which were the most important group. Dinoflagellates and coccolithophores occurred in fair numbers, the latter restricted to water of high salinity. Pennate diatoms were unexpectedly numerous inshore, most of them being bottom forms stirred up from below.

The chief feature of the Great Barrier Reef plankton is that there is no special seasonal maximum and minimum ; it is maintained at much the same level throughout the year. This is strikingly different from the conditions in temperate waters where one or two maxima constantly occur, the phytoplankton maximum being followed by a zooplankton maximum. Miss Marshall finds no real seasonal change in the composition of the diatom flora. Large increases

*British Museum (Natural History). Great Barrier Reef Expedition 1928-29. Scientife Reports. Vol. 2. No. 4. (a) "Variation in some Chemical Conditions on and near Low Isles Reef", (b) "The Temperature of the Waters in the Anchorage, Low Isles". (c) "Physical and Chemical Conditions in the Mangrove Swamps". By A. P. Or the Great Barrier Reef Region". By Sheina M. Marshall. 1933. sometimes occur, but not regularly. In the lagoon the wind keeps the water thoroughly mixed from top to bottom. The nutrient salts estimated were present only in very small quantities throughout the year and no relation was found between them and the diatom or dinoflagellate abundance. The type of plankton production found, the numbers low and varying little during the year, depends largely on physical conditions:

Trichodesmium occurs in large patches at irregular intervals, being most abundant in the calmer months from October to February. On August 22-25 large patches drifted on to the reef causing great distress to the fishes in the moats, and eventually decayed along the shore. In Mr. Orr's report (No. 4a), it is stated that at this time, because of Trichodesmium, although the water had been supersaturated with oxygen just when the tide left the reef flat, it was completely denuded of oxygen before the tide re-entered. Only pools in which the blue-green alga was deposited in quantity and decomposing showed this absence of oxygen. On no other occasion was a value below saturation with respect to oxygen found during the day on the flat.

The changes in temperature, salinity, oxygen and $p H$ value among the coral reefs are specially described and vary enormously, both daily and according to season. These changes may have an important effect on the reef organisms. Tidal and diurnal changes are on the whole more important than seasonal changes. The greatest differences occurred in coral pools isolated at low tide either during the night or during the day, the water being usually supersaturated with oxygen during the day and undersaturated at night.

In the mangrove swamps (No. $4 c$ ) the diurnal and seasonal changes are greatest at spring tides and are greater in summer than in winter. There are diurnal fluctuations in temperature, salinity, $p \mathrm{H}$ value and oxygen content in the mangrove swamps on Low Isles reef flat and usually a night fall and a day rise in temperature : salinity rises at low tide whilst $p \mathbf{H}$ value and oxygen saturation fall. 